\title{
Extinction during time controlled direct retinal stimulation after recovery from right hemispheric stroke
}

\author{
Richard F Kaplan, Ronald A Cohen, Axel Rosengart, Ann E Elsner, \\ Thomas R Hedges III, Louis R Caplan
}

\begin{abstract}
The temporal variables of extinction in two patients with right hemispheric stroke and two normal controls were studied using a scanning laser ophthalmoscope (SLO). This instrument enabled eye movements to be controlled for by projecting time controlled stimuli directly on to the retina. Both patients had recovered from their stroke (four months and five years) and seemed clinically normal. At $50 \mathrm{~ms}$, patient 1 extinguished one of two stimuli on seven of $\mathbf{4 0}$ double simultaneous stimulation (DSS) trials (five left, two right) and patient 2 extinguished the left stimulus on every DSS trial. At $100 \mathrm{~ms}$, patient 1 performed almost perfectly, whereas patient 2 extinguished on $30 \%$ of the DSS trials (10 left, two right). At $200 \mathrm{~ms}$, patient 2 performed perfectly. Neither of the controls showed extinction to DSS at any presentation time. Thus detecting DSS was time dependent in the patients but not the normal subjects. These data support the theory that recovery from neglect after right hemispheric damage may be mediated by the left hemisphere.
\end{abstract}

(F Neurol Neurosurg Psychiatry 1995;59:534-536)

Department of Neurology, Tufts
University School of

Medicine, Boston,

MA, USA

R F Kaplan

A Rosengart

$T$ R Hedges

L R Caplan

Department of

Behavioral Medicine,

Miriam Hospital,

Providence, RI, USA

R A Cohen

Schepens Eye Research Institute,

Boston, MA, USA

A E Elsner

Correspondence to: Dr Richard F Kaplan,

Department of Neurology,

New England Medical

Center, 750 Washington

Street, Boston, MA 02111 ,

USA.

Received 14 November 1995 and in final revised form

26 July 1995

Accepted 27 July 1995
Keywords: extinction; neglect; scanning laser ophthalmoscope

Extinction to double simultaneous stimulation (DSS) is a common manifestation of the neglect syndrome, ${ }^{1}$ although visual extinction and visuospatial neglect do vary indepenfails to report one of two simultaneously presented stimuli when each stimulus can be detected alone. As in other forms of neglect, extinction is more prevalent after right hemispheric damage. ${ }^{1-3}$

Poppelreuter ${ }^{4}$ provided one of the earliest descriptions of visual extinction, linking this phenomenon to an impairment of attention. Because visual acuity could be completely normal in the affected hemifield when only one stimulus was presented, he called the phenomenon a "hemianoptic weakness of attention." Critchley ${ }^{5}$ also considered extinction to be an attentional impairment, arguing dently. ${ }^{2}$ Extinction occurs when the patient that whereas imperception can be found together with inattention, inattention can occur without corresponding imperception. Other early investigators ${ }^{67}$ postulated, however, that attention alone could not account for visual extinction, proposing that extinction was also related to a perceptual imbalance between the intact and damaged hemispheres. Although a single stimulus in the affected visual hemifield can be perceived, its intensity is reduced or extinguished in relation to the perception of that stimulus in the intact field. Thus the normal process of interhemispheric rivalry was hypothesised as the basis for extinction, as the intact cortical hemisphere exerts dominance over the pathological hemisphere. ${ }^{7}$ Whereas hemispheric rivalry was used to describe differences in perception between the intact and damaged hemisphere, it has also become an explanation for hemispatial inattention. ${ }^{8}$ More recent investigations have shown that the probability of extinction can be manipulated by variables that affect both attention ${ }^{910}$ and perception. ${ }^{11}$

Because the extinction paradigm typically involves only two stimuli, it affords greater experimental control than other methods used to study spatial inattention. In the present study, we examined stimulus presentation time on extinction, while controlling spatial variables. Using a scanning laser ophthalmoscope (SLO), ${ }^{12}$ we were able to view the fundus and visually guide stimuli to precise retinal locations in real time. This enabled us to control for eye movements and project stimuli to each visual field while manipulating the duration of stimulus presentation.

\section{Case reports}

\section{PATIENT 1}

The patient, a 38 year old right handed man with no relevant history, awoke with left sided weakness, a facial droop, and confusion. On the previous day he noticed some problems ambulating, blurring of vision, and neck pain. On admission to New England Medical Center, he had a left hemiparesis with increased tone, dysarthria, left visual field neglect, motor impersistence, and prominent impulsivity. He was alert and oriented. Language and memory were normal. His visual fields were full but visual extinction to 
double simultaneous stimulation (DSS), using fingers, could not be assessed due to his lack of cooperation. Six days after the stroke onset, he continued to show neglect on letter cancellation but he was able to find the midpoint of a line and he did not extinguish to DSS. Brain MRI showed a right middle cerebral superior division infarction extending down to the horn of the lateral ventricle and out to the premotor and motor cortex. The basal ganglia and internal capsule were spared. Angiography and carotid ultrasound were consistent with a right internal carotid dissection. At the time of testing, four months after stroke, he showed no evidence of neglect on a letter cancellation test; nor did he extinguish during finger DSS.

\section{PATIENT 2}

The patient was a 49 year old right handed man who was admitted to hospital for acute onset of left sided weakness and slurred speech. He had a history of coronary artery disease and had undergone cardiopulmonary bypass surgery six weeks before this admission. The patient was alert and oriented. His speech was mildly dysarthric but otherwise normal. His memory was intact. Motor examination showed a slight hemiparesis. His sensory examination showed a prominent left sided astereognosis and agraphaesthesia. On bedside testing there was also visual extinction to DSS on the left. Reflexes were brisk bilaterally. The patient denied any neurological problems. Over the next two days, he developed a severe hemiparesis and left homonymous hemianopia. Computed tomography disclosed a large infarct in the distribution of the entire middle cerebral territory, involving frontal and parietal cortices, deep white matter, and basal ganglia. He was readmitted to the hospital eight months later after an apparent seizure and was subsequently maintained on tegretol. At the time of testing, five years after stroke, he had not had a seizure in three years. His visual fields were intact and he performed normally on a test of letter cancellation; however, he failed to report seeing the left finger on one of 10 visual DSS trials.

\section{CONTROL SUBJECTS}

Two normal subjects, a 48 year old right handed woman and a 63 year old left handed woman, with no history of neurological disease, were also tested for the purpose of comparison.

\section{DSS PROCEDURE}

A computer controlled SLO ${ }^{12}$ provided a high quality video image of the retina and generated the stimuli (figure). The timing was controlled by a Lafayette electronic tachistoscope attachment. A retinal field of $29 \times 23$ degrees was illuminated by a light of $830 \mathrm{~nm}$ which was invisible to the subject. Light stimuli of $633 \mathrm{~nm}$ at $0.1 \mathrm{~mW} / \mathrm{cm}^{2}$ forming the letter " $A$ " were 1.22 degrees high and 1.22 degrees wide. The difference in brightness between stimuli and background was $1.92 \mathrm{log}$ units. During the DSS trials, the two stimuli were 11.26 degrees apart and one was directed to either side of the fovea. The DSS trials were preceded by three randomly presented unilateral stimulation trials directed to either the right or left of the fovea. The stimuli were presented at 50,100 , or $200 \mathrm{~ms}$. Forty DSS trials were given at each presentation time. An equal number of DSS trials were preceded by three right unilateral trials, three left unilateral trials, a single right unilateral trial, and a single left unilateral trial. All stimuli were presented to the right eye.

\section{Results}

At $50 \mathrm{~ms}$, patient 1 correctly identified the location of all the unilateral stimuli, left and right. He extinguished one of the two stimuli, however, on seven of 40 DSS trials $(17.5 \%)$, five left and two right. He made no unilateral errors and extinguished the left stimulus on only one of 40 trials at $100 \mathrm{~ms}$. He was not tested at $200 \mathrm{~ms}$.

Patient 2 correctly identified unilaterally presented stimuli but extinguished the left sided stimulus on every DSS trial at $50 \mathrm{~ms}$. At $100 \mathrm{~ms}$, he extinguished one of two stimuli on 12 of 40 DSS trials (30\%), 10 left and two right. At $200 \mathrm{~ms}$, he performed perfectly on all DSS trials.
Photograph of the retina during DSS as viewed on the SLO monitor.

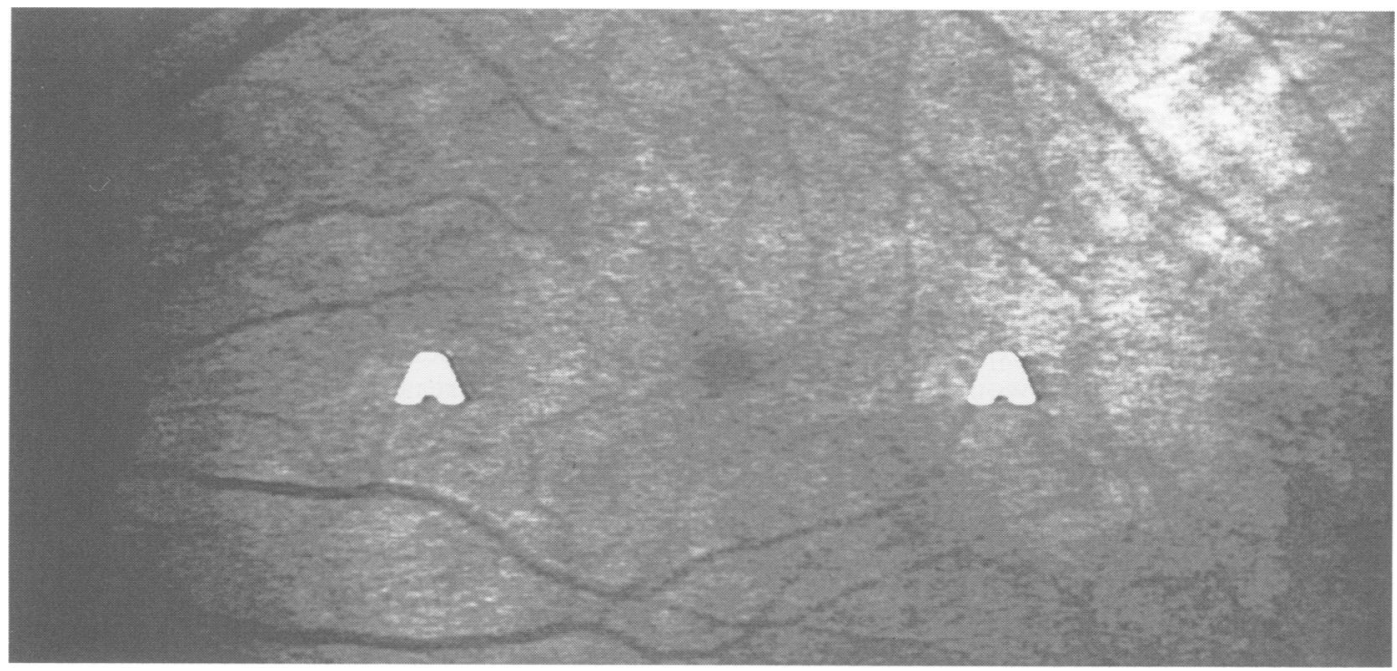


Both patients showed a decrease in the number of extinction errors across trials when we examined blocks of 10 successive trials. At $50 \mathrm{~ms}$, patient 1 made fewer left sided extinction errors during DSS after a right unilateral stimulus (two errors) compared with a left unilateral stimulus (three errors); whereas patient 2 made an equal number of left sided DSS extinction errors after right and left unilateral stimuli at $100 \mathrm{~ms}$.

Neither of the two controls showed extinction to DSS when the stimuli were presented at $50 \mathrm{~ms}$.

\section{Discussion}

The SLO provides a unique approach to the experimental study of visual neglect because time controlled stimuli can be directly projected onto the retina. ${ }^{12}$ Using this technique, residual deficits in extinction were elicited after apparent recovery in two patients at faster but not slower stimulus presentation times. Karnath ${ }^{9}$ also reported that whereas probability of extinction decreased a year after stroke, extinction persisted with very fast presentation times. Mattingley and coworkers $^{13}$ recently showed that although patients who had apparently recovered from right hemispheric damage may not show overt neglect, they continue to show an attentional bias towards ipsilateral space. These data, together with those of Karnath ${ }^{9}$, suggest that this is also the case in extinction. Furthermore, as stimuli in this study were always presented to the same retinal locations, improved detection required longer processing times as opposed to compensatory gaze shifts.

According to the limited attention theory, ${ }^{1}$ recovery after right hemispheric damage may be mediated by the intact left hemisphere. These data support that argument. Most theories $^{31415}$ of neglect are based on the premise that there is an asymmetry between the two cerebral hemispheres in mediating attention in space. Structural models ${ }^{14}{ }^{15}$ propose that the normal right hemisphere mediates attention towards both sides of space, whereas the left hemisphere normally mediates attention in contralateral space. Hence after right hemispheric damage, the patient shows a dramatic attentional bias toward the ipsilateral visual field, whereas after left hemispheric damage neglect is relatively rare. Implicit in these models is that the normal right hemisphere is capable of processing information from both sides of space in parallel. If after right hemispheric damage, the left hemisphere allocates its limited attentional resources to ipsilateral space, we would expect the present result - that is, extinction would be more likely to occur with decreased DSS presentation times. Furthermore this would suggest that the left hemisphere, unlike the right hemisphere, is limited by serial processing.
Lastly, we found that the probability of extinction, in both our patients, decreased inversely to the number of DSS trials. Poppelreuter $^{4}$ noted a similar phenomena which he ascribed to the patients adapting to the demands of the task. Normal subjects are capable of shifting visual attention to peripheral targets covertly, or independent of any overt shift in eye position. Patients with right parietal lobe damage, although no longer presenting with gross evidence of visual neglect, show deficits in covert orienting opposite to the side of their lesion. ${ }^{16}$ Whereas most patients with right hemispheric damage with neglect recover $^{17}$ and directional cuing has been implicated in recovery, ${ }^{1018}$ the mechanism by which neglect recovers has not been well studied. These data suggest that patients with right hemispheric damage can develop an ability to shift their attention in space without cuing or overt shifts in eye position. Further work may provide a better understanding of the natural history of recovery from neglect and aid in the development of rehabilitation strategies.

1 Heilman KM, Watson RT, Valenstein E. Neglect and related disorders. In: Heilman KM, Valenstein E, eds. Clinical neuropsychology. New York: Oxford University Press, 1993:279-336.

2 Vallar G, Rusconi ML, Bignamini L, Geminiani G, Perani D. Anatomical correlates of visual and tactile extinction in humans: a clinical CT study. $\mathcal{f}$ Neurol Neurosurg in humans: a clinical CT

3 Kinsbourne, M. Mechanisms of unilateral neglect. In: Jeannerod M, ed. Neurophysiological and neuropsychological aspects of spatial neglect. Amsterdam: Elsevier, cal aspects

4 Poppelreuter W. (translated by J Zihl) Disturbances of lower and higher visual capacities caused by occipital damage. Oxford: Clarendon Press, 1990.

5 Critchley $M$. The phenomenon of tactile inattention with special reference to parietal lesions. Brain 1949;72: 538-61.

6 Bender MB, Furlow LT. Phenomenon of visual extinction in homonymous fields and psychologic principles involved. Arch Neurol Psychiatry 1945;53:29-33.

7 Denny-Brown D, Meyer JS, Horenstein H. The significance of perceptual rivalry resulting from parietal lesions. Brain 1952;75:433-71.

8 Kinsbourne $M$. A model for the mechanism of unilateral neglect of space. Transactions of the American

9 Karnath H-O. Deficits of attention in acute and recovered visual hemi-neglect. Neuropsychologia 1988;26:27-43.

10 Kaplan RF, Verfaellie M, DeWitt LD, Caplan LR. Effects of changes in stimulus contingency on visual extinction. Neurology 1990;40:1299-301.

11 Farah MJ, Monheit MA, Wallace MA. Unconscious perception of "extinguished" visual stimuli: reassessing the evidence. Neuropsychologia 1991;29:949-58.

12 Elsner AE, Burns SA, Hughes GW, Webb RH. Reflectometry with a scanning laser ophthalmoscope. Applied Optics 1992;31:3697.

13 Mattingley JB, Bradshaw JL, Bradshaw JA, Nettleton AC Residual rightward attentional bias after apparent recovery from right hemisphere damage: implications for ery from right hemisphere damage: implications for a multicomponent model of neglect. $\mathcal{f}$ Neurol Neurosurg
Psychiatry 1994;57:597-604.

14 Heilman KM, Van DeAbell T. Right hemisphere dominance for attention: the mechanism underlying heminance for attention: the mechanism underlying hemi-
sphere asymmetries of inattention (neglect). Neurology sphere asymmetries

15 Weintraub S, Mesulam M-M. Right cerebral dominance in spatial attention. Arch Neurol 1987;44:621-25.

16 Posner MI, Walker JA, Friedrich FJ, Rafal RD. Effects of parietal injury on covert orienting of attention. $\mathcal{f}$ Neurosci 1984;4:1863-74.

17 Hier DB, Mondlock J, Caplan LR. Recovery of behavioral abnormalities after right hemisphere stroke. Neurology 1983;33:345-50.

18 Kaplan RF, Verfaellie M, Meadows M-E, Caplan LR, Pessin MS, DeWitt LD. Changing attentional demands in left hemispatial neglect. Arch Neurol 1991;48:1263-6. 